nature $P R E C E D I N G S 2009$

\title{
Chromosomal mutational algebra: a new algebra to manipulate chromosomal mutation
}

\section{Dipankar Mazumdar ${ }^{1}$}

\begin{abstract}
This study leads to a new algebra. An algebra is defined on the common mechanisms of chromosomal mutation. The algebra $<$ $S^{\Psi(\mathcal{C})}, *{ }^{\prime}, \Delta, D>$ is constructed for a given $\mathcal{C}$ and $\Psi$. This algebra represents the most common chromosomal mutational mechanisms. This can lead to a new way to manipulate chromosomal mutation with higher structures of abstract mathematics. The first proposal of the algebra was reported in the paper [1].
\end{abstract}

This study leads to a new algebra. An algebra is defined on the common mechanisms of chromosomal mutations. We first make the required segments of chromosome. This segmentation will be done depending on biological knowledge.

Define the segmentation $\Psi$ as, that $\Psi$ segments the chromosomal string $\mathcal{C}$. Particular biological knowledge will determine $\Psi$.

Consider an example. Let us suppose $\mathcal{C}$ is a chromosomal string comprising the neocleotides $a, t, g, c$ and

$$
\mathcal{C}=\text { atttgattacagatagaatagacagatagggatagacagat }
$$

A segmentation may be like,

\footnotetext{
${ }^{1}$ Department of Mathematics, Visva-Bharati, Santiniketan 731235, India. Email: itsmedip@rediffmail.com,dmazumda@science.uva.nl
} 


$$
\Psi(\mathcal{C})=\left\{\begin{array}{c}
\text { atttgattac } \\
\text { agatag } \\
\text { aat } \\
\text { agacagatagggat } \\
\text { agacagat }
\end{array}\right.
$$

Other segmentations can also be made depending upon the requirement.

Now we construct a set $S^{\Psi(\mathcal{C})}$ such that, $\varepsilon \in S^{\Psi(\mathcal{C})}, \varepsilon$ is the null element;

The segments returned by $\Psi(\mathcal{C}) \in S^{\Psi(\mathcal{C})}$ and if $x, y \in S^{\Psi(\mathcal{C})}$, then $x * y \in S^{\Psi(\mathcal{C})}$, the binary operation $*$ is the concatenation operation defined as usual.

For the set $S^{\Psi(\mathcal{C})}, \forall a, b \in S^{\Psi(\mathcal{C})}, a * b \in S^{\Psi(\mathcal{C})}$ by the definition of $S^{\Psi(\mathcal{C})}$.

Also $a *(b * c)=(a * b) * c$, clear from the operation of concatenation.

Thus $<S^{\Psi(\mathcal{C})}, *>$ forms a semigroup.

Thus we can conclude that,

Theorem. For a given $\mathcal{C}$ and $\Psi,<S^{\Psi(\mathcal{C})}, *>$ is a semigroup.

Now we see that,

i) $<S^{\Psi(\mathcal{C})}, *>$ is left cancellative.

i.e. $\forall a, b, c \in S^{\Psi(\mathcal{C})}, a * b=a * c \Rightarrow b=c$.

ii) $<S^{\Psi(\mathcal{C})}, *>$ is right cancellative.

i.e. $\forall a, b, c \in S^{\Psi(\mathcal{C})}, b * a=c * a \Rightarrow b=c$.

So, iii) $<S^{\Psi(\mathcal{C})}, *>$ is cancellative, as it is both left and right cancellative.

Theorem. The null element $\varepsilon$ satisfies the property of identity element $a * \varepsilon=\varepsilon * a=a, \forall a \in S^{\Psi(\mathcal{C})}$.

Proof follows from the definition of $\varepsilon$.

Theorem. For a given $\mathcal{C}$ and $\Psi,<S^{\Psi(\mathcal{C})}, *>$ is a semigroup with identity $\varepsilon$. So, $<S^{\Psi(\mathcal{C})}, *>$ is a monoid.

Now we consider a mutational mechanism - inversion. An inversion is a chromosomal mutation that results when a segment of a chromosome is excised and then reintegrated in an orientation of $180^{\circ}$ (degrees) from the original 
orientation.

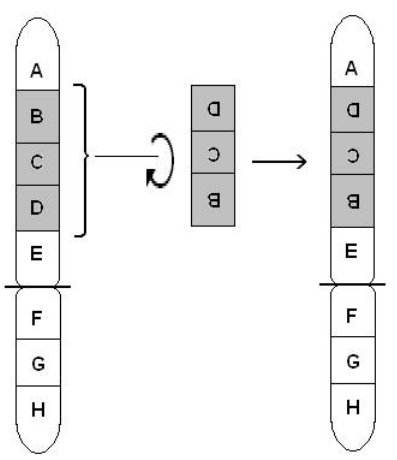

Figure 1: Inversion.

This inversion mechanism induce an operation involution.

Definition. The involution is a unary operation /: $S^{\Psi(\mathcal{C})} \rightarrow S^{\Psi(\mathcal{C})}$ such that $\forall a, b \in S^{\Psi(\mathcal{C})},\left(a^{\prime}\right)^{\prime}=a$ and $(a * b)^{\prime}=b^{\prime} * a^{\prime}$.

Thus it can be concluded that,

Theorem. For a given $\mathcal{C}$ and $\Psi,<S^{\Psi(\mathcal{C})}, *^{\prime},>$ is a monoid with involution.

Now we consider two chromosomal mutational mechanisms. Deletion and duplication.

A deletion is a mechanism involving the loss of a chromosomal segment.

A duplication is a mechanism that results in the doubling of a chromosomal segment.

Definition. Let us define the two unary operations induced by these two mutational mechanisms, over the monoid with involution $\left\langle S^{\Psi(\mathcal{C})}, *{ }^{\prime}>\right.$.

1. The deletion operation $\Delta: S^{\Psi(\mathcal{C})} \rightarrow\{\varepsilon\}$ as $\Delta(x)=\varepsilon, \forall x \in S^{\Psi(\mathcal{C})}$.

2. The duplication operation $D: S^{\Psi(\mathcal{C})} \rightarrow S^{\Psi(\mathcal{C})}$ as $D(x)=x * x, \forall x \in S^{\Psi(\mathcal{C})}$. 


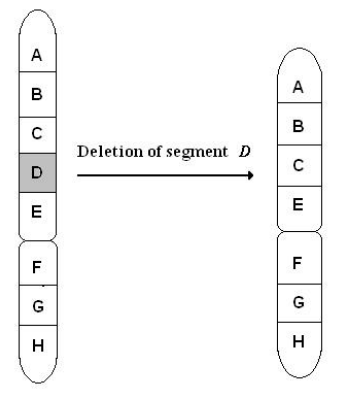

Figure 2: Deletion.

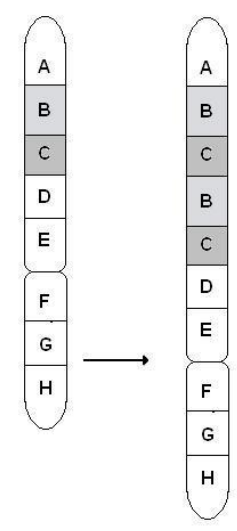

Figure 3: Duplication.

A new algebra $<S^{\Psi(\mathcal{C})}, *{ }^{\prime}, \Delta, D>$ is constructed for a given $\mathcal{C}$ and $\Psi$. This algebra represents the mentioned chromosomal mutational mechanisms. This can lead to a new way to manipulate chromosomal mutation with higher structures of abstract mathematics.

\section{References}

[1] Mazumdar, D., 2007, Chromosomal mutational algebra I, Advanced Modeling and Optimization, 9(2), 237-247.

[2] Russell, P.J., Genetics, Benjamin/Cummings, 1998. 